\title{
La sostenibilidad del sistema de pensiones en España
}

\author{
Luis-Jesús Belmonte ${ }^{1}$, Antonio-Jesús Corrales ${ }^{2}$ y Josefa Ruiz ${ }^{3}$ \\ ${ }^{1}$ Universidad de Almería (España) \\ 2 Ayuntamiento de Benahadux (España) \\ ${ }^{3}$ Hospital de Poniente (España)
}

\begin{abstract}
El objetivo de este trabajo consiste en presentar los importantes cambios demográficos que ha sufrido España en los últimos cincuenta años, marcados por un ineludible proceso de envejecimiento poblacional, que se ha contrarrestado tímidamente por la afluencia puntual de extranjeros. Aunque la garantía del sistema de pensiones es un hecho, durante las dos próximas décadas, hay que efectuar reformas con relativa urgencia, a imagen y semejanza de las ya realizadas en la mayoría de países europeos. La finalidad de estas reformas debe ser clara y decidida, pues es una certeza que en los próximos años se atenderá a un número mucho mayor de jubilados y no está suficientemente claro que se produzca un aumento proporcional en el número de afiliados. En cuanto al tipo de medidas a adoptar, creemos todas ellas deben de ir encaminadas a mejorar la relación entre las cotizaciones durante la etapa de actividad y la cuantía de las prestaciones, una vez que se produce la jubilación. Finalmente, sería deseable que toda reforma del sistema de pensiones fuese acompañada de medidas efectivas de política económica que incrementen el empleo, así como la calidad del mismo.
\end{abstract}

Palabras clave: Envejecimiento, reforma de las pensiones, edad de jubilación, tasa de dependencia.

The sustainability of the system of pensions in Spain. The aim of this work is to present the important demographic changes that Spain has experimented in the last 50 years, which are marked by an inescapable process of population aging. This fact has been minimized by the large number of foreigners who comes to Spain. Although the guarantee of the pension system is a fact, during the next two decades, it's needed to carry out some reforms with relative urgency, following the models that the most European countries have already carried out. The purpose of these reforms must be clear and precise, because it is a fact that in the following years will be a great number of retirees and is not so clear that a proportional increase in the number of participants will happen. With respect to the measures to be taken, we think that all of them must be directed to improve the relation between the contributions during the phase of activity and the amount of benefits, once retirement occurs. Finally, it would be desirable that any pension system's reform would be accompanied by effective measures of economic policy which increase employment, as well as its quality.

Key words: Ageing, pensions reform, retirement age, dependency ratio.

Correspondencia: Luis-Jesús Belmonte. Departamento de Economía Aplicada. Universidad de Almería. Ctra. La Cañada de San Urbano s/n, C.P. 04120. Almería (España). E-mail: 1belmont@ual.es 
A finales de 2008, coincidiendo con el estallido de la crisis financiera y su transmisión al resto de escenarios macroeconómicos, se volvió a plantear la problemática de la sostenibilidad del sistema actual de pensiones en España. En concreto, en octubre de 2008, a raíz de la presentación del Informe de Estabilidad de las Pensiones del Ministerio de Trabajo en Inmigración, se estableció un nuevo horizonte temporal, hasta el que la solvencia del sistema está garantizada: 2029.

Numerosos estudios previos habían fijado una fecha máxima de sostenibilidad del sistema de pensiones español y numerosas alternativas (Devesa y Devesa, 2008; Jiménez-Ridruejo, 2008; Alonso y Conde-Ruiz, 2007; Balmaseda, Blázquez, Martín-Moreno y Tello 2006; Jimeno, 2003; Barea, 2000; Conesa y Garriga, 2000; Eguia, 1997). Incluso el propio Ministerio de Trabajo e Inmigración, en el año 2002, estableció que la solvencia del sistema estaba garantizada hasta 2015 (Ministerio de Trabajo y Asuntos Sociales, 2001). Afortunadamente, los cambios en las previsiones han sido favorables, por cuanto que se amplían las garantías de las prestaciones, aunque también induce a pensar que una coyuntura atípica desde el punto de vista económico podría acortar dicho plazo garantizado. En este sentido, con objeto de sortear la situación actual de crisis económica, se han propuesto una serie de medidas que, en algunos casos, podrían mermar el fondo de reserva de las pensiones, si no se estudian suficientemente sus efectos, como expondremos más adelante.

Como cualquier otra previsión, con el paso de los meses, la realidad económica nacional se ocupará de desmentir o de refrendar la fecha fin de sostenibilidad del sistema. En este sentido, lo más importante no debe ser acertar en la fecha límite de una quiebra hipotética, sino el análisis de la situación, actual y futura, que dará lugar a ese desequilibrio, con objeto de implementar las medidas necesarias para evitarlo.

Así pues, este trabajo comienza con un breve análisis demográfico, en el que se presta una atención especial al aumento de la población de edad avanzada, así como sus principales consecuencias. Posteriormente, en el tercer apartado y cuarto apartado, se presenta una breve radiografía de la protección social en Europa y en España, respectivamente, haciendo hincapié en la estrecha relación que ésta guarda con el ciclo económico. Finalmente, se exponen las principales conclusiones de este trabajo, así como las medidas que, a corto y medio plazo, el Gobierno tendrá que llevar a cabo.

\section{TENDENCIA DEMOGRAFICA EN ESPAÑA}

Desde el punto de vista económico, la base demográfica de un país se constituye como el primer eslabón del futuro crecimiento económico, por cuanto que el número de efectivos laborales, su capital humano y la duración de la jornada laboral 
influyen de forma decisiva a la hora de garantizar las pensiones del futuro, aunque de forma bien distinta.

En concreto, la financiación del sistema de pensiones se obtiene de las cotizaciones a la Seguridad Social, es decir, está relacionada directamente con el número de afiliados al sistema. Sin embargo, la calidad de las prestaciones, es decir, su cuantía y su estabilidad, dependen del grado de competitividad de la mano de obra nacional, con relación al resto de países con los que compite comercialmente. De ahí la necesidad de incrementar la productividad de la mano de obra nacional, es decir, de la calidad de la hora efectiva de trabajo incorporada al proceso productivo, con objeto de conseguir incrementar la competitividad de la economía española.

Así pues, en la medida en que trabajen afiliadas más personas en España, con un nivel de productividad y horario adecuados, más y mejor será la cantidad de servicios totales de trabajo que se podrá incorporar al proceso productivo con el fin de producir bienes y servicios (Ruesga et al., 2002). Desafortunadamente, en los últimos años, la cantidad de servicios totales de trabajo que ha disfrutado el mercado laboral español se ha caracterizado por la intensidad en el empleo y no por su calidad/productividad.

Por otra parte, si hasta ahora se ha considerado la importancia del volumen de afiliados a la Seguridad Social y la calidad de sus prestaciones, no se debe de olvidar la importancia creciente que están adquiriendo los grupos de edad avanzada, a partir de 65 años, que constituyen el grueso del colectivo en el que se aplica el gasto de las cotizaciones. En este sentido, si bien es cierto que se ha producido un importante aumento de la esperanza de vida, la relevancia que adquiere la tercera edad se ve reforzada por otros factores, tales como: el descenso de la natalidad, la disminución de la tasa de fecundidad e importantes cambios en los hábitos sociales.

Figura 1. Evolución de la población de edad avanzada (1900 - 2010)

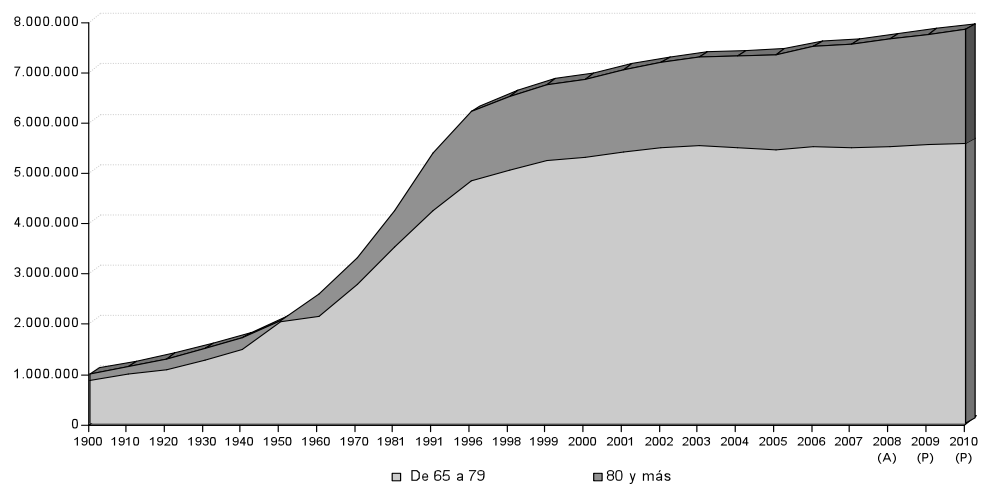

Fuente: INE. Elaboración propia. 
La cuestión del envejecimiento de la población afecta a la mayoría de países desarrollados y más industrializados del mundo, como es el caso europeo (Whiteford y Whitehouse, 2006). A diferencia de otras regiones del mundo, como es el caso de África y Asia, las bajas tasas de natalidad, fecundidad y mortalidad están favoreciendo el crecimiento de la población más longeva.

En el caso de España, no se pueden obviar los efectos económicos de un colectivo de más de siete millones de personas, que se materializan en unos costes cada vez mayores de la dependencia económica, sanitaria y social, con cargo a la Seguridad Social o de los familiares, a la vez que puede convertirse en un importante yacimiento de yacimiento de empleo, siempre y cuando se despejen las dudas de la financiación de tales coberturas.

A diferencia de lo que ocurría en la primera mitad del siglo XX, con la consolidación del estado de bienestar en España, el colectivo de población de edad avanzada ha ido ganando peso sobre el total poblacional, especialmente en las tres últimas décadas. Es necesario destacar que se trata de un dato muy positivo, aunque también es cierto que el aumento de los mayores ha venido acompañado de:

- El empeoramiento de la tasa de natalidad y fecundidad, que unido a la disminución de la tasa de mortalidad, ha contribuido a potenciar el envejecimiento de la población española.

- Incremento de la esperanza de vida de nuestros mayores, que se ha traducido en un aumento considerable de los años de disfrute de la vejez. Este hecho será decisivo a la hora de considerar la reforma del sistema actual de pensiones, como se describirá más adelante. Asimismo, se incrementa la necesidad de potenciar las actividades de ocio con las que contentar a una parte de los jubilados, los más jóvenes (65 - 75 años), y en plenas facultades físicas y mentales.

- Pérdida de atención hacia los mayores en el seno familiar, como consecuencia de la creciente incorporación de la mujer al mercado de trabajo. Al mismo tiempo, como consecuencia de la baja tasa de fecundidad, los hogares tienen cada vez menos descendientes y además son más activos desde el punto de vista laboral.

- Incremento sin precedentes de la población extranjera, especialmente a partir del año 2000, que ha venido a contrarrestar la escasa natalidad y fecundidad de la población autóctona, al tiempo que ha contribuido a elevar las cotizaciones a la Seguridad Social y a mejorar la ratio afiliados por cada jubilado (Figura 2).

Sin embargo, a finales de 2008 el impacto de la crisis provocó una fuerte reducción del número de afiliados (850.000), que ha tenido especial incidencia sobre la población extranjera. Es por ello que, en los próximos dos años, se prevé una caída de la ratio de afiliados por cada jubilado hasta niveles de comienzos de 2000, con el riesgo que este hecho puede entrañar a la hora de garantizar la sostenibilidad de las pensiones.

Desde el punto de vista de la evolución demográfica de España, resulta evidente el cambio sustancial que ha sufrido la pirámide de población española en las 
últimas décadas. En este sentido, la convergencia económica con la Europa más desarrollada también llevó aparejada la similitud en las pautas de crecimiento poblacional. Así pues, desde 1960, cuando existía un perfil de población joven, con altas tasas de natalidad y escaso peso de la población avanzada, se ha pasado a una situación que se caracteriza por la forma ovalada de la pirámide, con un vértice cada vez más amplio (Figura 3).

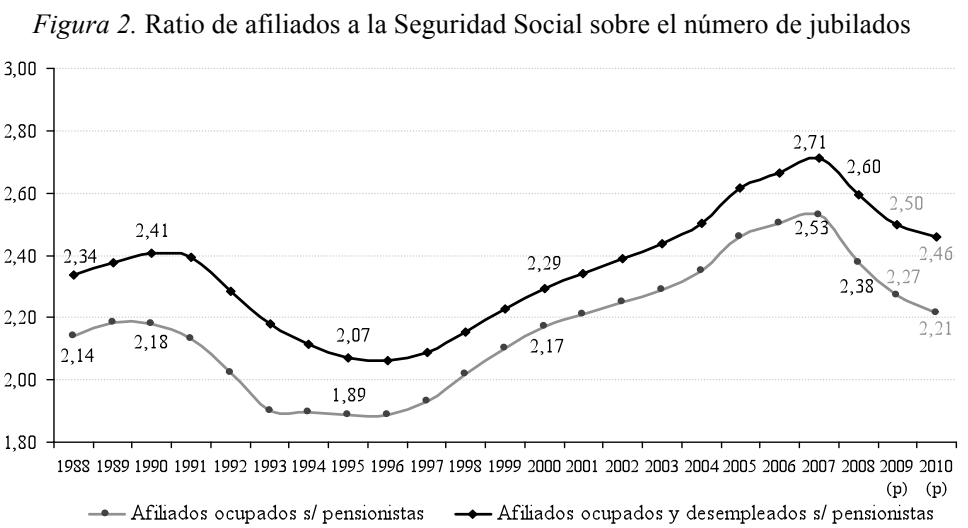

Fuente: Ministerio de Trabajo e Inmigración. Elaboración propia.

Figura 3. Evolución de la pirámide de población española (1960, 1981, 1998 y 2008)
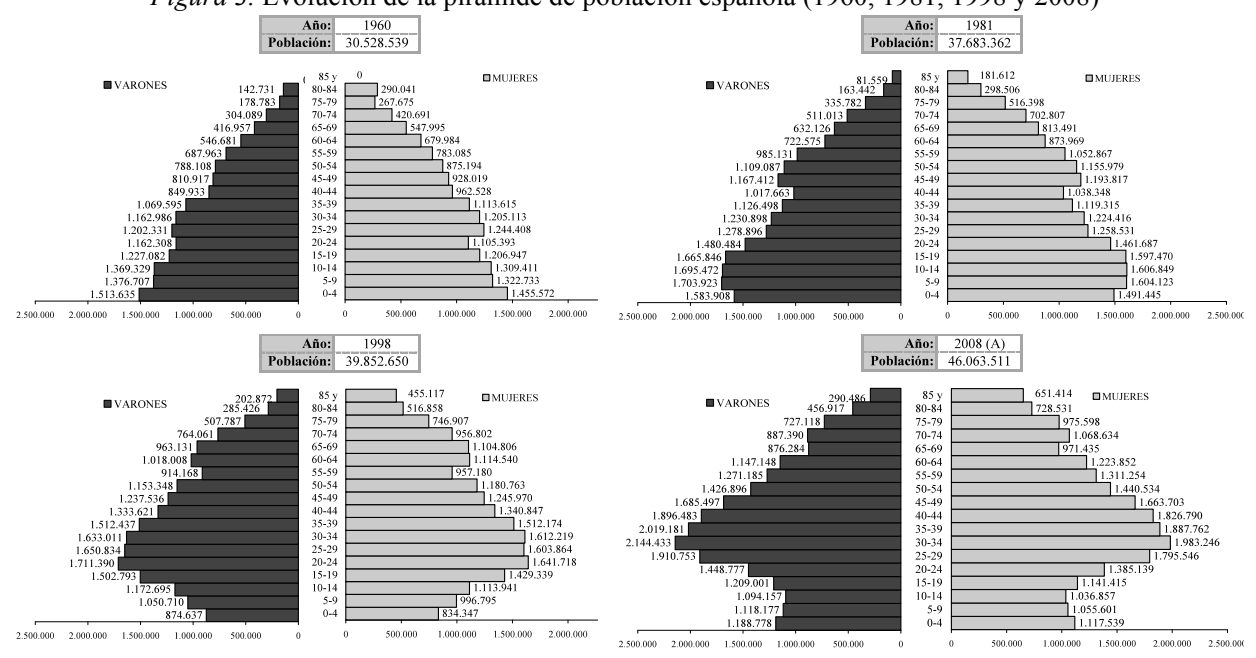

Fuente: INE. Elaboración propia. 
En valor absoluto, en los últimos años, la población mayor no ha dejado de incrementarse, especialmente el tramo correspondiente a los mayores de 80 años, pero lo más importante es que también lo ha hecho su proporción sobre el total poblacional. En concreto, si en 1960 este colectivo representaba el 1,42\% del total de la población, en 1981 el 1,92\%, en 1998 este porcentaje casi se duplicaba (3,66\%) y, diez años más tarde, se situaba en el 4,62\%. Por su parte, en 2008, la población mayor de 65 años representó el 16,57\% de la población total, casi el doble que en 1960, cuando este colectivo representaba sólo el $8,41 \%$ del total.

Finalmente, a partir de 2000, se observa como la inmigración en masa ha facilitado el estiramiento de los grupos quinquenales centrales (20-64 años), es decir, el perfil edad más abundante en este colectivo (Aznar et al., 2009; p. 20). Sin embargo, en la medida en que se mantenga la actual situación de crisis económica, deberían de aplicarse los principios de la movilidad geográfica de la mano de obra, es decir, que la disminución de las oportunidades laborales en el territorio nacional puede provocar la migración a otros países, más atractivos desde el punto de vista económico (Borjas, Freeman y Katz, 1996; Viaña, 1996 y Tapiños, 1996).

\section{LA SITUACION EN EUROPA: LA TASA DE DEPENDENCIA Y EL NIVEL DE BIENESTAR SOCIAL}

Es un hecho que el avance que está experimentando el colectivo de población de edad avanzada tendrá importantes implicaciones socioeconómicas, tanto para las actuales, como para las generaciones venideras. Se trata de un proceso demográfico que afecta a la vieja Europa y que tendrá consecuencias claras en:

- El incremento del gasto público aplicado a la protección social, ya sea como pensiones contributivas o como no contributivas, así como el resto de prestaciones que otorgan los gobiernos y que conforman el estado del bienestar de un país. A este respecto, es más que probable que el incremento en el volumen de las prestaciones por habitante vaya acompañado del aumento en el número de beneficiarios, con lo que el impacto en las finanzas públicas será doble.

En el caso de España, será preciso incrementar el volumen de atenciones y cuidados a los mayores, garantizando por Ley el cuidado personal de este colectivo. En este sentido, hay casi dos millones de mayores dependientes, de los cuales algo más de doscientos mil viven en hogares colectivos.

- Se producirá un aumento considerable en los gastos sanitarios, tanto por el número de efectivos a tratar, como por el incremento de las dolencias que presentará un colectivo de pacientes con una edad cada vez más avanzada, entre los que se hacen cada vez más comunes enfermedades como el Alzheimer y demás trastornos mentales (Gázquez et al. 2008). 
Se producirá un incremento de la demanda de actividades culturales y de ocio en el colectivo de edad avanzada, que será especialmente importante en el tramo inicial de su jubilación (65-75 años). En promedio, conservarán la plenitud de sus facultades físicas y mentales, así como la capacidad económica suficiente para no menospreciar el impacto de este nuevo yacimiento de empleo.

Desde el punto de vista cuantitativo, en los próximos 50 años se producirá un incremento considerable de la tasa de dependencia, entendida ésta como la relación entre el número de personas mayores de 65 años por cada 100 personas en edad de trabajar (15-64 años). Resulta destacable observar la extraordinaria evolución de esta ratio en todos los países europeos (Tabla 1).

\begin{tabular}{|c|c|c|c|c|c|c|c|c|}
\hline \multirow[b]{2}{*}{ País } & \multicolumn{7}{|c|}{ Evolución de la tasa de dependencia } & \multirow{2}{*}{$\begin{array}{c}\text { Esperanza } \\
\text { de vida } \\
2006\end{array}$} \\
\hline & 2010 & 2020 & 2030 & 2040 & 2050 & 2060 & $\Delta 10 / 60$ & \\
\hline Eslovaquia & 17,0 & 23,9 & 32,3 & 40,0 & 55,5 & 68,5 & $304,1 \%$ & 74,4 \\
\hline Polonia & 19,0 & 27,2 & 36,0 & 41,3 & 55,7 & 69,0 & $263,4 \%$ & 75,3 \\
\hline Rumanía & 21,3 & 25,7 & 30,3 & 40,8 & 54,0 & 65,3 & $205,9 \%$ & 72,6 \\
\hline Lituania & 23,2 & 26,0 & 34,7 & 42,8 & 51,1 & 65,7 & $183,2 \%$ & 71,1 \\
\hline Rep. Checa & 21,8 & 31,1 & 35,7 & 42,7 & 54,8 & 61,4 & $181,3 \%$ & 76,8 \\
\hline Malta & 21,2 & 31,3 & 39,1 & 41,7 & 49,8 & 59,1 & $178,8 \%$ & 79,5 \\
\hline Irlanda & 16,7 & 20,2 & 24,6 & 30,6 & 40,4 & 43,6 & $161,4 \%$ & 79,7 \\
\hline Eslovenia & 23,9 & 31,2 & 40,8 & 49,4 & 59,4 & 62,2 & $160,1 \%$ & 78,3 \\
\hline Letonia & 25,2 & 28,1 & 34,6 & 40,7 & 51,2 & 64,5 & $156,1 \%$ & 70,9 \\
\hline Bulgaria & 25,3 & 31,1 & 36,3 & 43,6 & 55,4 & 63,5 & $151,2 \%$ & 72,7 \\
\hline Chipre & 18,0 & 22,3 & 27,4 & 30,8 & 37,7 & 44,5 & $147,1 \%$ & 80,6 \\
\hline España & 24,4 & 27,4 & 34,3 & 46,4 & 58,7 & 59,1 & $141,8 \%$ & 81,1 \\
\hline Hungria & 24,2 & 30,3 & 34,1 & 40,1 & 50,8 & 57,6 & $138,0 \%$ & 73,5 \\
\hline Estonia & 25,0 & 29,2 & 34,4 & 39,0 & 47,2 & 55,6 & $122,1 \%$ & 73,1 \\
\hline Holanda & 22,8 & 30,7 & 40,0 & 46,8 & 45,6 & 47,2 & $106,7 \%$ & 80,0 \\
\hline Europa [27] & 25,9 & 31,1 & 38,0 & 45,4 & 50,4 & 53,5 & $106,4 \%$ & $:$ \\
\hline Portugal & 26,6 & 30,7 & 36,6 & 44,6 & 53,0 & 54,8 & $106,0 \%$ & 78,9 \\
\hline Grecia & 28,2 & 32,8 & 38,5 & 48,3 & 57,0 & 57,1 & $102,4 \%$ & 79,5 \\
\hline Austria & 26,0 & 29,2 & 38,1 & 46,0 & 48,3 & 50,7 & $94,7 \%$ & 80,1 \\
\hline Finlandia & 25,7 & 36,8 & 43,9 & 45,1 & 46,6 & 49,3 & $91,8 \%$ & 79,6 \\
\hline Italia & 31,0 & 35,5 & 42,5 & 54,1 & 59,2 & 59,3 & $91,4 \%$ & : \\
\hline Alemania & 31,2 & 35,3 & 46,2 & 54,7 & 56,4 & 59,1 & $89,5 \%$ & 79,9 \\
\hline Luxemburgo & 21,1 & 24,2 & 30,8 & 36,3 & 37,8 & 39,1 & $85,6 \%$ & 79,4 \\
\hline Bélgica & 26,1 & 30,6 & 37,6 & 42,3 & 43,9 & 45,8 & $75,7 \%$ & 79,5 \\
\hline Francia & 25,8 & 32,8 & 39,0 & 44,0 & 44,7 & 45,2 & $75,1 \%$ & 80,9 \\
\hline Dinamarca & 25,0 & 31,9 & 37,9 & 42,7 & 41,3 & 42,7 & $70,8 \%$ & 78,4 \\
\hline Reino Unido & 24,7 & 28,6 & 33,2 & 36,9 & 38,0 & 42,1 & $70,5 \%$ & $:$ \\
\hline Suecia & 27,8 & 33,7 & 37,4 & 40,8 & 41,9 & 46,7 & $68,0 \%$ & 81,0 \\
\hline
\end{tabular}

Fuente: Eurostat. Elaboración propia. 
En los próximos años, tal y como se indica en el informe de la Comisión Europea (Comisión Europea, 1996; Ploug, 2003) y en contra de la evidencia actual, se prevé un considerable empeoramiento de la tasa de dependencia en los países del Este de Europa: Eslovaquia, Polonia, Rumania, Lituania, República Checa, Eslovenia,... que, además de ostentar la mayor tasa de dependencia prevista para 2060, son los que más aumentan en el horizonte 2010-2060. Por el contrario, los países que actualmente presentan las mayores ratios de dependencia, fundamentalmente las grandes economías europeas, incrementarán moderadamente su tasa en los próximos cincuenta años. Es el caso de Suecia, Reino Unido, Dinamarca, Francia, Bélgica, Luxemburgo, Alemania,... que sufrirán un incremento de la dependencia inferior a la media de la Unión Europea de $\operatorname{los} 27$.

De manera particular, resulta sorprendente la evolución demográfica que presentará Francia en los próximos cincuenta años, es decir, la más que moderada evolución de la dependencia. Es por ello que podría tomarse este ejemplo como muy válido a la hora de establecer una efectiva política de mejora de la tasa de natalidad, vía prestaciones familiares adecuadas (Villa y López, 2008).

Es muy posible que las proyecciones de Eurostat se vean matizadas en los próximos años, en el caso en que los flujos migratorios se inviertan, es decir, los países de la vieja Europa reciban cada vez menos inmigrantes, pues permanezcan en sus países emisores. Así pues, el rejuvenecimiento demográfico que supone una población inmigrante puede ser transitorio, tanto por la inversión del flujo migratorio, como por la previsible integración social en los países de acogida, por lo que disminuirá la tasa de fecundidad de este colectivo al mimetizarse con la población autóctona.

Asimismo, en algunos casos, no se encuentra suficientemente estimado el efecto llamada que puede causar en los países de origen el estado del bienestar que se disfruta en las economías más desarrolladas, por lo que no se puede prever un flujo de migraciones duradero (Giorgi y Pellizzari, 2009).

Irremediablemente, el envejecimiento de la población europea requerirá un mayor esfuerzo presupuestario para dar cobertura a las pensiones del futuro. Sin embargo, en los últimos años se ha asistido a la estabilización del gasto público en protección social, tanto en lo que concierne al gasto per cápita, como en porcentaje de PIB. Es el caso de Alemania, Dinamarca, Francia e, incluso, España.

Técnicamente, lo que se está produciendo es una limitación del gasto en protección social, optando por las reformas de los sistemas de prestaciones como vía más rápida para afrontar los próximos retos demográficos (Kemmerling y Neugart, 2009, Herce, 1997). En este sentido, con objeto de diluir el gasto en pensiones, muchos países de la Unión Europea han iniciado cambios sustanciales en su actual sistema de pensiones. Es el caso de Dinamarca, que ampliará la edad de jubilación desde los 65 a los 67 años, entre 2024 y 2027; Alemania, que ha adoptado la misma medida, aunque 
de manera más gradual (entre 2012 y 2029) y Reino Unido, que ampliará su edad de jubilación desde los 65 a los 68 años, con el horizonte de 2024 a 2046. Parece lógico que la ampliación de la edad de jubilación será algo muy común en los principales sistemas de pensiones europeos, en vista del incremento de la esperanza de vida.

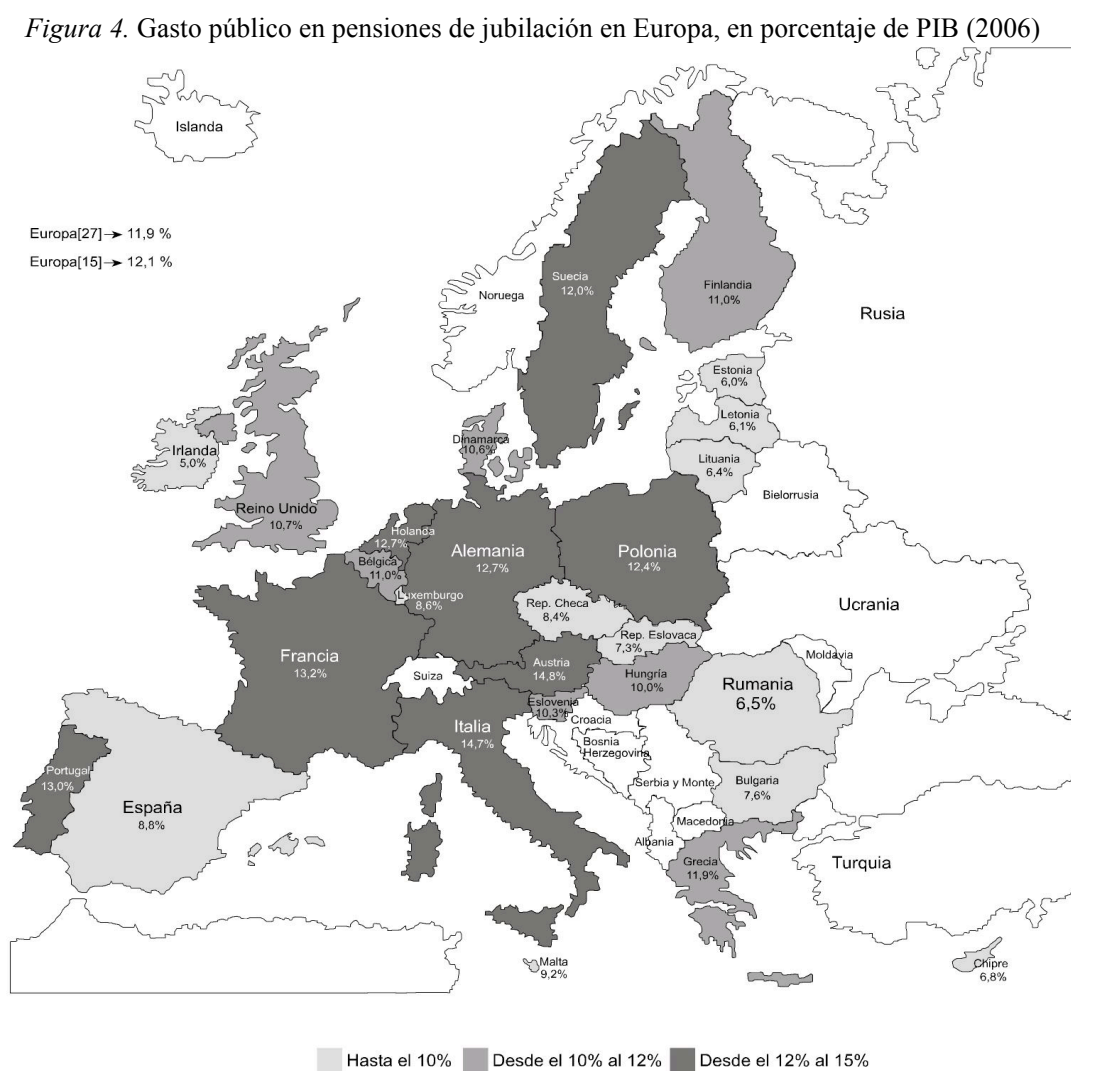

El gasto público en protección social incluye cualquier tipo de gasto relacionado con la lucha contra la exclusión social, ayudas familiares, ayudas para el alojamiento, pensiones, gastos generados por el desempleo y parte de los gastos en sanidad. Asimismo, incluye todos aquellos gastos necesarios de administración y funcionamiento asignables a este tipo de prestaciones. Asimismo, el gasto público dedicado a pensiones incluye todos aquellos fondos que se destinan al pago de pensiones por varios motivos: de jubilación (parcial o total, anticipadas o no, contributivas o no contributivas), de invalidez, así como todas aquellas prestaciones de prejubilación, cualquiera que sea su causa.

Fuente: Eurostat. Elaboración propia.

\section{EVOLUCION DE LA PROTECCION SOCIAL EN ESPAÑA}

La protección social en España tiene su máximo exponente en nuestro sistema público de pensiones, que ha sido objeto de numerosas reformas, entre las que 
destaca la que se realizó en 1995: el Pacto de Toledo. En aquel momento, se hizo necesaria la adopción de un conjunto de medidas que garantizaron la sostenibilidad del sistema, tales como la ampliación de la base reguladora y el aumento de la edad legal de jubilación. Paralelamente, estas medidas tenderían a contener el importante gasto social, que se estaba generando con las pensiones. Afortunadamente, la garantía del sistema se ha logrado gracias a:

- La etapa de bonanza económica que se inició a finales de los noventa, que provocaría un incremento sostenido y continuado del número de cotizantes (ingresos para la Seguridad Social), a lo que contribuyó activamente la afluencia de inmigrantes.

- El modesto crecimiento del número de jubilados, pues las últimas cohortes de jubilados han pertenecido a los nacidos durante la Guerra Civil, por lo que el gasto en pensiones ha sido menos que proporcional al crecimiento de la afiliación.

Hasta ahora, la organización de las pensiones en España gira en torno a un sistema de reparto, es decir, aquel en el que los que están cotizando a la Seguridad Social (afiliados) financian las pensiones de los que actualmente están jubilados. Además, se trata de un sistema público que se articula en dos subsistemas de pensiones: el contributivo y el no contributivo (Figura 5):

- El sistema de pensiones contributivas engloba al conjunto de prestaciones económicas, normalmente de carácter indefinido, que requieren la acreditación de un periodo mínimo de cotización y cuya cuantía, con carácter general, está en función de varios parámetros:

o La cuantía de las cotizaciones que haya efectuado el candidato a la jubilación, así como el empresario si se trabaja por cuenta ajena, durante el periodo de cotización. Es lo que constituye la base reguladora de la pensión.

o Poseer una edad mínima de jubilación de 65 años, aunque se permite la jubilación anticipada a partir de los 60 años, con la consecuente reducción en el importe de la pensión a percibir.

o Contar con un periodo mínimo de cotización de 15 años, de los que los dos últimos serán inmediatamente anteriores al momento de la jubilación. En este sentido, cuanto mayor sea el número de años cotizados, menor será el coeficiente reductor que multiplicará a la base reguladora (suma de cotizaciones de los últimos 15 años). De tal manera que, con 35 ó más años cotizados, se percibirá el $100 \%$ de la base reguladora.

A su vez, el sistema público de pensiones contributivas se subdivide en aquel que recoge las prestaciones de los funcionarios (denominado sistema de Clases Pasivas) y el que engloba al resto de trabajadores (que se nutre con las cotizaciones a la Seguridad Social que hacen los trabajadores y los empresarios).

- El sistema no contributivo se diferencia del anterior, en que no se dota con las cotizaciones de trabajadores ni empresarios, sino a través de impuestos generales. En este caso, los beneficiarios suelen ser personas pertenecientes a colectivos en riesgo 
de exclusión, que no tienen por qué alcanzar los 65 años de dad, ni contar con un periodo mínimo de cotización.

Desde el punto de vista económico, casi el 95\% del presupuesto total de pensiones se emplea en prestaciones contributivas, mientras que sólo algo más de un $5 \%$ se dedica a pensiones no contributivas. Hace una década, las pensiones contributivas suponían poco más del $90 \%$, mientras que las no contributivas se acercaban al $10 \%$. Afortunadamente, la buena evolución de la economía nacional ha hecho que aumente la primacía de las prestaciones que se financian con las cotizaciones a la Seguridad Social.

Figura 5. Tipos de pensiones en España (2008)

\section{Total pensiones}

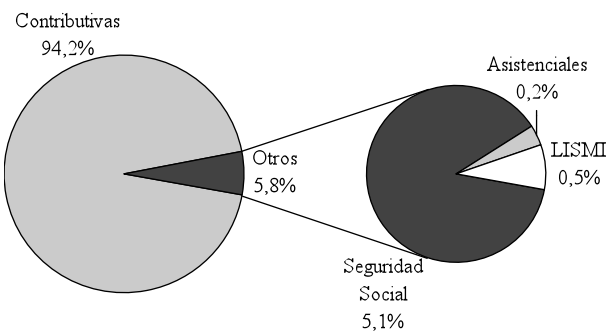

Pensiones contributivas

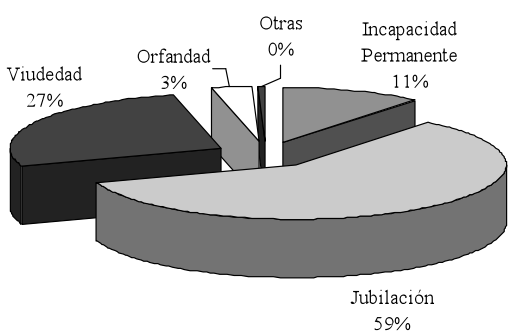

Fuente: Ministerio de Trabajo e Inmigración. Elaboración propia.

Por otra parte, del total de prestaciones contributivas, las pensiones de jubilación representan el capítulo más importante, con casi el $60 \%$ del total. Le siguen las pensiones de viudedad y de incapacidad permanente, aunque a bastante distancia (Figura 5).

En la figura 6 se muestra cómo la situación de superávit (eje principal) se relaciona estrechamente con la evolución del empleo/afiliación (eje secundario), que es el principal inductor de los ingresos de la Seguridad Social, vía cotizaciones. Desde 1995, la afiliación ha pasado de poco más de doce millones de personas a algo más de diecinueve millones en 2007. El efecto sobre la contabilidad de la Seguridad Social fue decisivo para desterrar el déficit que arrastraba el sistema con anterioridad a 1999. Asimismo, hay que destacar la moderada evolución del número de jubilados en los últimos años, por lo que apenas se ha restado impulso al efecto positivo que supone un incremento del volumen de cotizaciones.

Tras observar la estrecha relación entre el déficit/superávit público, así como el de la Seguridad Social, con el volumen de afiliados, resulta oportuno considerar que, en los próximos años, se hará más patente la imposibilidad de financiar el sistema de pensiones con la única aportación de las cotizaciones. De hecho, el auxilio gubernamental mediante aportaciones extraordinarias se hará cada vez más necesario, a 
medida que aumente el peso de los jubilados sobre el total de cotizantes y la crisis económica se agudice, a no ser que se efectúen reformas en el sistema público de pensiones.

Figura 6. Evolución del Déficit/Superávit Público, del saldo y de la afiliación a la Seguridad Social $(1995-2010)$
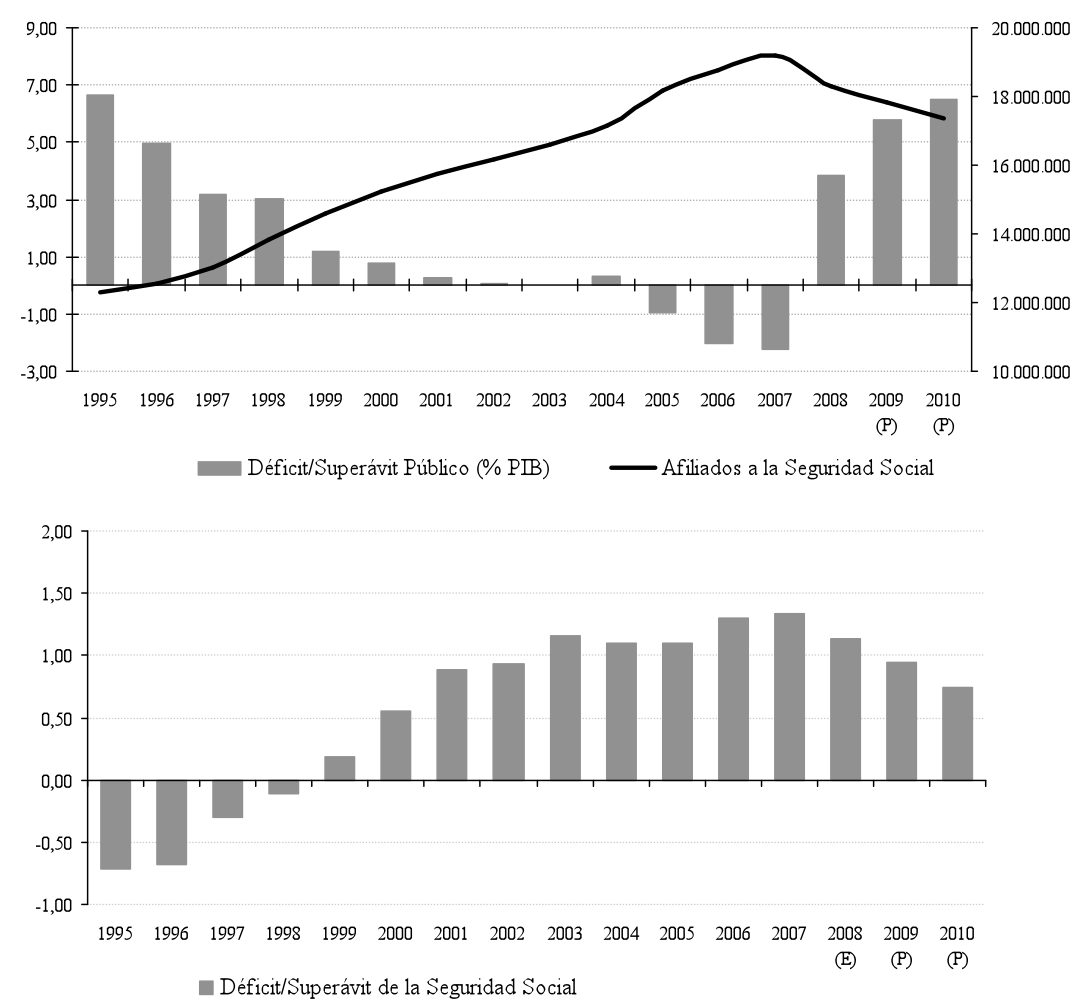

Fuente: Ministerio de Trabajo e Inmigración. Elaboración propia.

Afortunadamente, en el caso español y en el de la mayoría de sistemas de pensiones de reparto, se cuenta con el apoyo financiero de los denominados fondos de reserva, con los que hacer frente a una mala coyuntura económica, que provoque la reducción de la base de cotizantes. En ausencia de la contribución estatal por haber constituido este fondo, sería necesario aumentar las cuotas de cotización a la Seguridad Social, a empresas y a trabajadores, con las que evitar recortes significativos en las prestaciones, puesto que las jubilaciones se otorgan bajo la premisa de un sistema de prestación definida. Sin embargo, es previsible que los jubilados del futuro se vean 
afectados por posibles recortes debido a la generosidad de nuestro actual sistema (Alonso y Conde-Ruiz, 2007; Monereo, 2008).

Es por ello que, de cara al futuro, existe cierto consenso académico y político sobre la necesidad de efectuar una reforma del actual sistema de pensiones, según las proyecciones de población que se manejan para los próximos años. A este respecto, las previsiones del Ministerio de Trabajo e Inmigración (2008a) indican que el sistema público de pensiones español está garantizado hasta 2023, aunque si se hace uso del Fondo de Reserva para las pensiones dicha garantía podría extenderse hasta 2029. Afortunadamente, los años de bonanza económica han retrasado en ocho años la desestabilización del sistema, ya que, con anterioridad, en la previsión realizada en 2005, el sistema estaba garantizado sólo hasta 2015.

A modo de esquema, se pueden agrupar las propuestas que actualmente se barajan, en:

- Ampliación de los años para el cálculo de la base de cotización. Se trataría de modificar las bases y algoritmos de cálculo de las prestaciones con la finalidad de ajustarlas cada vez más a las cotizaciones que las originan. La idea consistiría en limitar cada vez más el impacto de la redistribución, con objeto de aumentar la proporcionalidad entre el periodo de cotización y el de disfrute de la pensión. En concreto, se piensa en ampliar aún más el periodo de cálculo de las pensiones, así como el número de años necesarios para tener derecho a las mismas. Esta medida ya se usó en la reforma de las pensiones de 1985 cuando se elevó el período mínimo de cotización para acceder a una pensión de jubilación de diez a quince años. Asimismo, se amplió el periodo de cómputo de las cotizaciones, para calcular el importe de la pensión, de dos a ocho años (Mota, 2006; p. 36).

- Favorecer la fiscalidad de los instrumentos de ahorro/previsión. Independientemente de cual sea el desenlace de las futuras reformas de las pensiones, se deben de incrementar los incentivos para que se contraten productos de ahorro para la jubilación. En este sentido, aunque en el caso de España, el Ministerio de Trabajo e Inmigración indica que la garantía se extiende hasta 2029, la aversión al riesgo que emana del concepto de jubilación debe incitar a los trabajadores a diversificar su futuro, complementando la posible prestación pública con otras fórmulas de ahorro/previsión, siempre que se prime su contratación desde el punto de vista fiscal.

- Incentivar la base de afiliados a la Seguridad Social. Se trata de una propuesta surgida a comienzos de 2009, a iniciativa del Servicio de Estudios del BBVA. Esta medida plantea la rebaja en las cotizaciones sociales como vía para abaratar la contratación e incentivar el empleo. En este sentido, hay que tener en cuenta que las rebajas en las cotizaciones inciden directamente en el saldo de la Seguridad Social, por lo que hay que asegurarse de que una disminución en los tipos de cotización va a ser compensada con un incremento del número de afiliados. En caso contrario, si la rebaja en las cotizaciones no incentiva el empleo en términos netos el efecto podría ser doblemente negativo: a la vez que baja el número de cotizantes lo haría la 
recaudación del colectivo de trabajadores que renueve periódicamente su prestación laboral.

- Incrementar la edad media de jubilación. En concreto, con los últimos datos disponibles del Ministerio de Trabajo e Inmigración, la edad media de jubilación es de algo más de 63 años, es decir, dos años por debajo de la edad legal de jubilación (65 años). Asimismo, existe un alto ritmo de jubilaciones anteriores a los 65 años, un $42 \%$ de los nuevos jubilados (Ministerio de Trabajo e Inmigración, 2008b), mientras que el $57 \%$ se jubila con 65 o más años.

Adicionalmente, desde un punto de vista laboral, sólo un $1,5 \%$ de los mayores de 65 años permanece como activo, principalmente en el sector servicios y por cuenta propia. Cada vez es más frecuente la jubilación precoz a los 60 años, incluso antes, a pesar del coeficiente reductor que se aplica en el cálculo de la pensión. Es por ello que, en el futuro, es previsible que se acometa una reforma del actual sistema de pensiones, para hacerlo más acorde al periodo de disfrute de la pensión, es decir, mejorar el principio de contributividad, ya que el problema está en cómo financiar las pensiones del futuro. Por ahora, se incentiva el retraso de la jubilación con un $2 \%$ por cada año que exceda de 65 años.

Finalmente, en cualquier caso, el sistema resultante tendrá que cumplir con las dos funciones básicas que se exigen a todo sistema público de pensiones, es decir:

- Que posea unas prestaciones dignas que salvaguarden de la pobreza a todos los mayores.

- Que cuenten con la diversidad adecuada para contribuir al mantenimiento de los niveles de renta disponible anteriores a la jubilación.

\section{CONCLUSIONES}

En el futuro, el análisis de la población de edad avanzada será una cuestión a tener en consideración de cara a la planificación del gasto social, así como de la dotación de servicios asistenciales, en vistas de la creciente dependencia que presentará este colectivo.

Así pues, desde el punto de vista presupuestario, es posible que se decida replantear el actual sistema de pensiones, toda vez que el número de cotizantes se reduce y el de beneficiarios se amplía. Si en la actualidad la cobertura de casi 8 millones de jubilados está generando ciertas dudas respecto a la financiación, la situación será especialmente preocupante si la crisis económica persiste y además el número de pensionistas de duplica en los próximos cuarenta años, a la vista de la evolución de la tasa de dependencia.

A pesar de ello, es muy cierto que las generaciones que se jubilarán en los próximos 20-25 años han tenido más facilidad para diversificar su futuro, pues han podido ahorrar más (especialmente en bienes inmuebles), a la vez que poseen planes de 
pensiones privados. Sin embargo, en un horizonte mayor, los jóvenes que ahora tienen serios problemas para encontrar un empleo estable, cuando lo encuentran lo hacen a una edad mayor y carecen de estabilidad en sus ingresos, pueden tener problemas para acceder a una cobertura similar a la que disfrutan nuestros mayores en la actualidad. A muchos de ellos les parecerá una utopía constituir una base de cotización superior a los 35 años, que es la que da derecho a la percepción del $100 \%$ de la pensión.

Adicionalmente, en la próxima década se producirá el pase a la jubilación de todos los nacidos en los años sesenta, es decir, durante el baby boom, con lo que el incremento de perceptores de pensiones de jubilación se incrementará de manera abrupta, previsiblemente.

Es por ello que, con la debida antelación, es posible que se adopten medidas para incentivar el retraso en la edad de jubilación, con la finalidad garantizar la solvencia del sistema y dar tiempo a la constitución de una base de cotización adecuada, que también habría que ampliar. Asimismo, a medio plazo también es factible la idea de ir sustituyendo el actual proceso de acumulación de 20-25 años de ocio posteriores a la jubilación, por un disfrute continuado a lo largo de la vida activa, fomentando la jubilación parcial o incluso el retraso en edades más tardías, cercanas a los setenta años, en vistas de la evolución de la esperanza de vida.

Por otra parte, creemos que la solución al sostenimiento del actual sistema de pensiones no está en una nueva reglamentación laboral, sino en la ganancia de competitividad de nuestra economía. Un reto que se logra con el incremento de la productividad, es decir, con la innovación tecnológica y organizativa, así como con el necesario incremento del capital humano. Es la única manera para que el empleo sea de calidad y, lo más importante, éste exista.

Finalmente, sería aconsejable que se apoyase aún más la recuperación de las tasas de fecundidad, para acercarla y mantenerla en niveles próximos a la tasa de reemplazo generacional, con lo que se conseguiría diluir el peso de la tercera edad, a la vez que se limita la dependencia de la inmigración como vía para mantener el crecimiento poblacional. En este sentido, será esencial abordar la necesaria conciliación de la vida familiar y laboral pues, a priori, podría considerarse como contradictorio desear que aumente la tasa de fecundidad y, al mismo tiempo, se incremente la tasa de actividad femenina, alejada en más de veinte puntos porcentuales de la masculina. No es algo utópico o imposible, pues en otros países, como en Francia, se ha logrado.

\section{REFERENCIAS}

Alonso, J. y Conde-Ruiz, J.I. (2007). Reforma de las pensiones: la experiencia internacional. Información Comercial Española, 837, 179-193. 
Aznar, J.A., Belmonte, L.J., Bonillo, D., Molina, A. y Saquilán, H. (2008). Análisis de la inmigración en la provincia de Almería. Junta de Andalucía-Consejería de Gobernación. Delegación de Gobierno de Almería.

Balmaseda, M., Blázquez, J., Martín-Moreno, J.M. y Tello, P. (2006). Efecto de cambios legislativos en las pensiones de jubilación contributivas: el caso español. El Trimestre Económico, 73, 611-637.

Barea, J. (2000). Una solución para la viabilidad financiera del sistema de pensiones contributivas. Hacienda Pública Española, (monográfico), 9-20.

Borjas, G.J., Freeman, R.B. \& Katz, L.F. (1996). Searching for the effect of immigration on the labor market. The American Economic Review, 86 (2), 246-252.

Conesa, J.C. y Garriga, C. (2000). Reforma del sistema de seguridad social y adquisición de formación. Investigaciones Económicas, XXIV(2), 271-295.

Comisión Europea (1996). The impact of ageing on public expenditure: projections for the EU25 member states on pensions, health care, long-term care, education and unemployment transfers (2004-2050). Comisión Europea, Bruselas.

Giorgi, G. \& Pellizzari, M. (2009). Welfare migration in Europe. Labour Economics, 886, (inpress).

Devesa, J.E. y Devesa, M. (2008). Desequilibrio financiero-actuarial en el sistema de pensiones de jubilación del régimen general. Revista de Economía Aplicada, 16, 85-117.

Eguía, B. (1997). Seguridad Social y estructura demográfica en un modelo de ciclo vital con edad de retiro endógena. Revista de Economía Aplicada, 15, 5-38.

Gázquez, J.J., Pérez, M.C, Lucas, F. y Yuste, N. (2008). Prevalencia de los trastornos mentales en la población mayor. Anales de Psicología, 24(2), 327-333.

Herce, J.A. (1997). La reforma de las pensiones en España: aspectos analíticos y aplicados. Moneda y Crédito, 204, 105.143.

Jiménez-Ridruejo, Z. (2008). Envejecimiento e inmigración: consecuencias sobre la sostenibilidad del sistema de pensiones en España. Revista del Ministerio de Trabajo y Asuntos Sociales, extra, 359-377.

Jimeno, J.F. (2003). La equidad intrageneracional de los sistemas de pensiones. Revista de Economía Aplicada, 33, 5-48.

Kemmerling, A. \& Neugart, M. (2009). Financial market lobbies and pension reform. European Journal of Political Economy, 25, 163-173.

Ministerio de Trabajo e Inmigración (2008a). Estrategia Nacional de Pensiones. Secretaría de Estado de la Seguridad Social del Ministerio de Trabajo y Asuntos Sociales, Madrid. Disponible en: http://www.tt.mtas.es/periodico/seguridadsocial/200810/ SS20081023_2.htm

Ministerio de Trabajo e Inmigración (2008b). Anuario de Estadísticas del Ministerio de Trabajo e Inmigración 2007. Subsecretaría de Trabajo e Inmigración, Madrid. Disponible en: http://www.mtin.es/estadisticas/ANUARIO2007/welcome.htm

Ministerio de Trabajo y Asuntos Sociales (2001). Proyección económico-actuarial del gasto en pensiones contributivas del sistema de la seguridad social y evolución prevista de las cotizaciones del sistema. Documento para la comisión no permanente para la valoración 
de los resultados obtenidos por la aplicación de las recomendaciones del Pacto de Toledo. Dirección General de Ordenación Económica de la Seguridad Social, Madrid.

Monereo, J.L. (2008). Ciclos vitales y Seguridad Social: trabajo y protección social en una realidad cambiante. Revista del Ministerio de Trabajo y Asuntos Sociales, extra, 49-134.

Mota, R. (2006). La política socialista de pensiones de jubilación (1982-1996): entre gradualismo y redistribución. Panorama Social, 4, 32-45.

Ploug, N. (2003). Recalibración del sistema de pensiones de vejez en Dinamarca. Revista internacional de Seguridad Social, 2, 77-96.

Ruesga, S., Lasierra, J. M. y Murayana, C. (2002). Economía del Trabajo y Política Laboral. Madrid: Ediciones Pirámide.

Tapiños, G.P. (1996). Las migraciones internacionales y el desarrollo. Estudios Migratorios, 2, 9-37.

Viaña, E. (1996). Migraciones en el Mediterráneo occidental: población, economía y sociedad. Revista Asturiana de Economía, 5, 193-206.

Villa, L.E. y López, L. (2008). Jubilación y prolongación de la vida activa (las mil y una jubilaciones). Revista del Ministerio de Trabajo y Asuntos Sociales, extra, 379-403.

Whiteford, P. \& Whitehouse, E. (2006). Pension challenges and pension reforms in OECD countries. Oxford Review of Economic Policy, 22, 78-94.

Recibido: 2 de enero de 2009

Modificaciones: 7 de mayo de 2009

Aceptado: 11 de mayo de 2009 